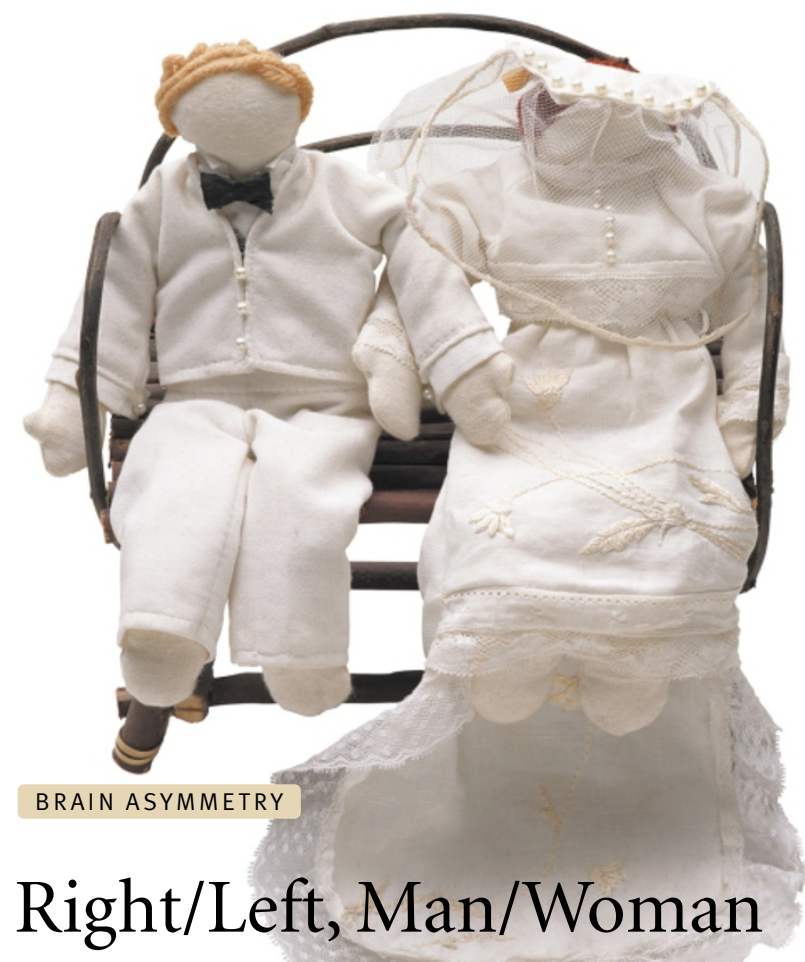

It's a well-known fact that the nervous system is asymmetric; we have been aware of this organizational principle ever since Broca's classic studies on aphasia in the nineteenth century. In fact, brain asymmetry is arguably the neurobiological concept best known by the general public, epitomized by the study of split-brain patients and the 'left/verbal, right/abstract' dichotomy that pervades popular culture. It's also well known that the brain is sexually dimorphic; some brain structures are different in males and females, and a lot of research has been devoted to understanding the functional consequences of this dimorphism. Now, Cahill et al. have put an interesting twist on this cerebral Yin/Yang by showing that memory formation for emotional stimuli leads to the asymmetric activation of the amygdala, activation that differs markedly between men and women.

The authors used positron-emission tomography to image brain glucose activity while subjects viewed a series of emotionally charged or neutral films, and asked the volunteers to rate their emotional reaction to each clip. They found that viewing emotive films was associated with increased activity in the amygdala, which correlated with a better recollection of the emotionally charged clips three weeks later. However, the activation was not bilateral but showed strong asymmetry; only the amygdala of one hemisphere was engaged by the task. But surprisingly, the activated side differed between the sexes - the right amygdala was activated in men and the left amygdala in women.

It is not clear what the gender differences and the asymmetric activation found in this study might mean. As there seemed to be a slight variation in the specific region of the amygdala activated in men and women, the differences might actually translate into the activation of very dissimilar neural circuits in each case. But regardless of their meaning, the findings of Cahill and his colleagues add to the emerging list of gender-related functional asymmetries, an essentially unexplored territory of brain function.

Juan Carlos López

(2) References and links

ORIGINAL RESEARCH PAPER Cahill, L. et al. Sex-related difference in amygdala activity during emotionally influenced memory storage. Neurobiol. Learn. Mem. 75, 1-9 (2001) FURTHER READING Cahill, L. in The Amygdala: A Functional Analysis (ed. Aggleton, J) 425-444 (Oxford Univ. Press, Oxford, 2000) | Gazzaniga, M. S. Principles of human brain organization derived from split-brain studies. Neuron 14, 217-228 (1995) ENCYCLOPEDIA OF LIFE SCIENCES Brain imaging: localization of brain functions | Limbic system

H I G H L I G H T S

DEPRESSION

\section{The more the merrier}

Our understanding of depression has come a long way since Hippocrates' proposal that melancholia was due to an excess of the 'bodily humour' black bile. But, although a range of therapies for depression are now available, the underlying molecular and cellular mechanisms of their action are not well understood.

Several studies have indicated that depression might be linked to stressinduced degeneration and loss of neurons in the hippocampus. Interestingly, the hippocampus is one of the brain regions in which new neurons are produced throughout life in adult animals, including humans. So is it possible that fluctuations in the number of neurons in the hippocampus could be an important factor in the onset of and recovery from depression? Malberg and colleagues began to test this idea by determining the influence of various antidepressant treatments on hippocampal neurogenesis.

Neurogenesis in the hippocampus of adult rats after chronic antidepressant treatment was followed using the thymidine analogue bromodeoxyuridine (BrdU), which acts as a marker for proliferating and newly born cells. Several long-lasting antidepressant treatments were tested, including fluoxetine (Prozac) and electroconvulsive seizure (ECS), which is clinically the most effective treatment for refractory depression. All of the treatments significantly increased the number of BrdU-positive cells in the hippocampus relative to the control, with ECS ( $~ 50 \%$ increase) being more effective than the chemical antidepressants (20-40\% increase). Additionally, acute treatment with fluoxetine did not have this effect, which is consistent with the time course for its therapeutic action.

Neurogenesis could be regulated at several stages, including cell proliferation, differentiation and survival. The authors showed that chronic antidepressant treatment increased the number of BrdU-positive cells owing to an increase in cell proliferation and that

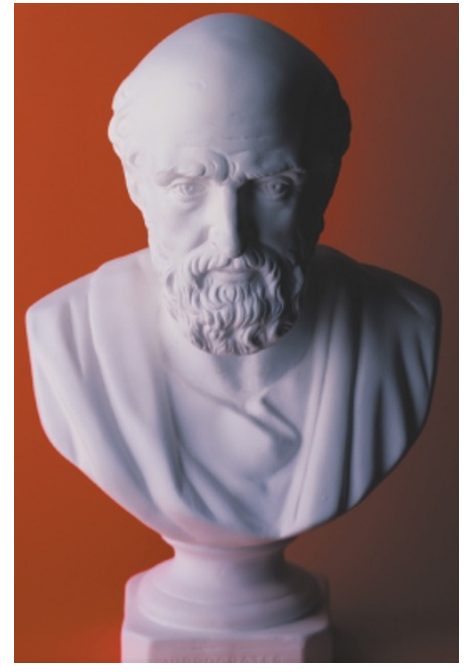

Hippocrates (460-377 BC). @ Ed Eckstein/CORBIS.

the antidepressant treatment did not directly affect the rate of cell maturation and survival. But what becomes of the newly born cells? To answer this question, Malberg et al. determined the number and phenotype of the BrdU-positive cells once they had matured. At this time, the number of BrdU-positive cells was still significantly increased in the animals that had received antidepressant treatment. Immunofluorescent labelling of the BrdU-positive cells with neuronal and glial markers revealed that most of the surviving BrdU-positive cells were neuronal rather than glial. So, the increased cell proliferation induced by antidepressant treatment results in a net increase in hippocampal neurons.

Although it is too early to say whether the growth of new brain cells is relevant to the action of antidepressants in humans, these results seem to be an important part of the story. Current research is investigating the molecular mechanisms involved in the upregulation of cell proliferation in response to antidepressant treatment, as better understanding of these processes might lead to more effective drugs to combat depression.

Peter Kirkpatrick

6) References and links ORIGINAL RESEARCH PAPER Malberg, J. E. et al. Chronic antidepressant treatment increases neurogenesis in adult rat hippocampus.

J. Neurosci. 20, 9104-9110 (2000)

J. Neurosci. 20, 9104-9110 (2000)
FURTHER READING Jacobs, B. L. et al. Adult brain neurogenesis and psychiatry: a novel theory of depression. Mol. Psychiatry 5, 262-269 (2000) Duman R. S. et al Neuronal plasticity and suniva in mood disords. Biol. Psychiaty 48, 732-739 in (2000) | Gross, C. G. Neurogenesis in the adult brain: death of a dogma. Nature Rev. Neurosci. 1 , 67-73 (2000) 